\title{
Position finding using simple Doppler sensors
}

\author{
S. Schelkshorn and J. Detlefsen \\ Technische Universität München \\ Lehrstuhl für Hochfrequenztechnik \\ Fachgebiet Hochfrequente Felder und Schaltungen \\ 80333 München, Germany
}

\section{Basic principle}

In the case of a single doppler sensor at a fixed position one can only measure the velocity, i.e. the change in distance, of an moving object relative to the fixed sensor. This relative velocity is simply the projection of the velocity vector on the directional vector from the sensor to the object. Even if the objects position is known the velocity vector can not be determined as an infinite number of velocity vectors has the same projection (Fig. 1a shows two possibilities).

Adding a second doppler sensor at another fixed position to this system it is possible to combine the two relative velocities and the knowledge of the two directional vectors to obtain the correct velocity vector of the moving object (Fig. 1b).

If on the other hand the position of the object is unknown but the velocity vector is known, any number of object positions result in the same measured relative velocity at a single sensor. Adding again a second sensor now the unknown position can be found (see Fig. 2).

Combining the two preceeding examples one ends up with the case where neither the objects position nor its velocity is known. Both properties can be calculated from the relative distance information acquired by four doppler sensors.

\section{Solving the system of nonlinear equations}

Each of the four sensors measures the change in distance $\delta d^{i, j}$ from its location $\boldsymbol{x}_{\mathrm{B}}^{i}$ with $i=1 \ldots 4$ to the object $\boldsymbol{x}_{\mathrm{P}}^{j}$.

$\delta d^{i, j}=\left|\boldsymbol{x}_{\mathrm{P}}^{j}(k T)+\boldsymbol{v}_{\mathrm{P}}^{j}(k T)-\boldsymbol{x}_{\mathrm{B}}^{i}\right|-\left|\boldsymbol{x}_{\mathrm{P}}^{j}(k T)-\boldsymbol{x}_{\mathrm{B}}^{i}\right|$

The measured values of all four sensors can be used to setup a system of equations for the unknowns. This system is a nonlinear system of equations.

A well known method for solving a system of nonlinear equations is the so-called Newtons Method (Werner, 1992). This method is based on the iterative solution of a linearized

(simon.schelkshorn@tum.de)

Published by Copernicus Publications on behalf of the URSI Landesausschuss in der Bundesrepublik Deutschland e.V. 

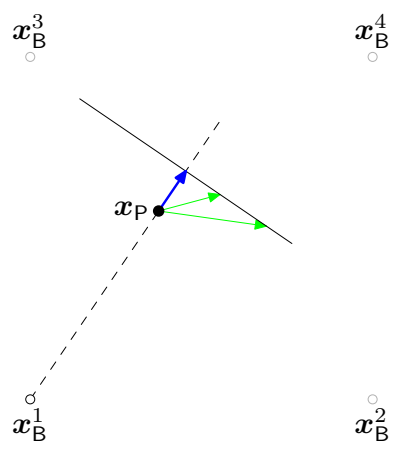

(a) Multiple possibilities for the velocity vector when using only one sensor

Fig. 1. Velocity measurement using one or two doppler sensors, position $x_{P}$ is known.

version of the system of equations. Linearization is done at assumed values $\boldsymbol{b}_{0}^{\ell}$ for the unknowns. The linearized change in distance can be written as follows

$\delta d^{i, j}(\Delta)=\delta d_{0}^{i, j, \ell}+a^{i} \cdot \delta x_{0}^{\ell}+b^{i} \cdot \delta y_{0}^{\ell}+c^{i} \cdot \delta v_{x 0}^{\ell}+d^{i} \cdot \delta v_{y 0}^{\ell}$

where $a^{i} \ldots d^{i}$ are the according taylor coefficients. Combining all four sensors using this linearized expression above yields

$$
\left[\begin{array}{llll}
a^{1} & b^{1} & c^{1} & d^{1} \\
a^{2} & b^{2} & c^{2} & d^{2} \\
a^{3} & b^{3} & c^{3} & d^{3} \\
a^{4} & b^{4} & c^{4} & d^{4}
\end{array}\right] \cdot\left[\begin{array}{l}
\delta x_{0}^{\ell} \\
\delta y_{0}^{\ell} \\
\delta v_{x 0}^{\ell} \\
\delta v_{y 0}^{\ell}
\end{array}\right]=\left[\begin{array}{l}
\delta d^{1, j}(\Delta) \\
\delta d^{2, j}(\Delta) \\
\delta d^{3, j}(\Delta) \\
\delta d^{4, j}(\Delta)
\end{array}\right]-\left[\begin{array}{l}
\delta d_{0}^{1, j, \ell} \\
\delta d_{0}^{2, j, \ell} \\
\delta d_{0}^{3, j, \ell} \\
\delta d_{0}^{4, j, \ell}
\end{array}\right]
$$

Equation (3) can also be written in a short form.

$\boldsymbol{S}_{0}^{\ell} \cdot \delta \boldsymbol{b}_{0}^{\ell}=\left(\delta \boldsymbol{d}^{i, j}(\Delta)-\delta \boldsymbol{d}_{0}^{i, j, \ell}\right)$

$\delta \boldsymbol{b}_{0}^{\ell}$ represents the deviation of the unknowns from the assumed values $\boldsymbol{b}_{0}^{\ell}$. It is calculated by inverting $\boldsymbol{S}_{0}^{\ell}$.

$\delta \boldsymbol{b}_{\mathrm{opt}}^{\ell}=\left[\boldsymbol{S}_{0}^{\ell \mathrm{T}} \boldsymbol{S}_{0}^{\ell}\right]^{-1} \boldsymbol{S}_{0}^{\ell \mathrm{T}} \cdot\left(\delta \boldsymbol{d}^{i, j}(\Delta)-\delta \boldsymbol{d}_{0}^{i, j, \ell}\right)$

For the next iteration $\boldsymbol{b}_{0}^{\ell}$ is improved by $\delta \boldsymbol{b}_{0}^{\ell}$.

$\boldsymbol{b}_{0}^{\ell+1}=\boldsymbol{b}_{0}^{\ell}+\delta \boldsymbol{b}_{0}^{\ell}$

This procedure is continued until convergence is reached.

When solving a system of nonlinear equations by using its linear couterpart one has to choose an initial value for the unkowns. If this guess lies close enough to the solution the iterative solution process will converge to the correct values (Hettwer and Benning, 2001). If the initial guess is an unsuitable one the iterative solution may diverge or in the worst case converge to a wrong value. In the case of divergence one can simply choose a different set of initial values and try again but in the case of convergence to a wrong result it is
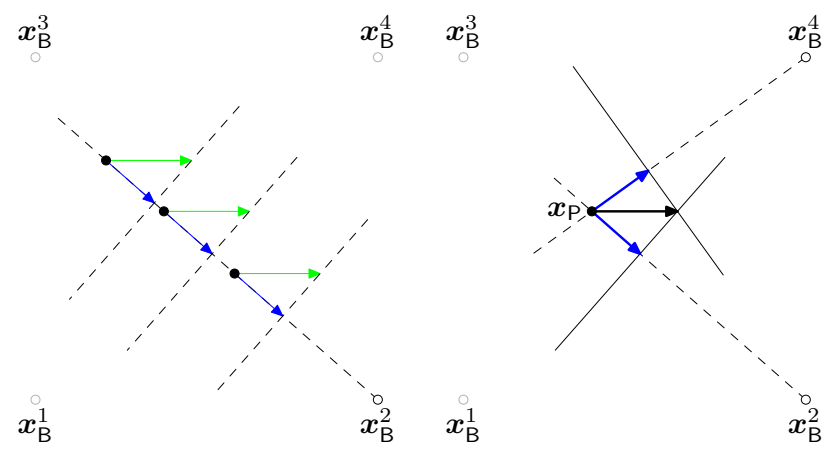

(a) Possible object positions when using only one sensor.

(b) Determining the correct position using two sensors.

Fig. 2. Position finding using one or two doppler sensors, velocity $v_{\mathrm{P}}$ is known.

impossible to detect. So choosing the right initial value for the linearization turns out to be the key issue in our problem.

In our case we have no information on the properties of the unknown object, so choosing an appropriate initial value for the linearization is difficult. Therefore it would be very helpful if the number of possible initial values that achieve convergence can be increased.

In the following four different methods for solving our system of equations are to be compared. The first as well as the simplest is the standard Newtons Method. The three additional methods are all based on it.

The second method is called Newton Method with additional attenuation (Hettwer and Benning, 2001). It differs from the standard version only in the way how the improved values are computed from the initial ones. Here $\delta \boldsymbol{b}_{0}^{\ell}$ is multiplied by an attenuation factor before it is added to $\boldsymbol{b}_{0}^{\ell}$.

$\boldsymbol{b}_{0}^{\ell+1}=\boldsymbol{b}_{0}^{\ell}+\lambda^{\ell} \delta \boldsymbol{b}_{0}^{\ell}$

The attenuation factor $\lambda^{\ell} \leq 1$ is chosen individually for each iteration. The selection is based on a residual that is a measure for the difference between the linearized and the nonlinear system of equations.

Both methods mentioned so far utilize the measured change in distance during one timestep $T$. To take advantage of the measurement at two consecutive timesteps, a second timestep can be incorporated in Eq. (3) as four additional equations thus leading to an overdetermined system of equations. This extension can be done for both methods, the standard Newtons Method and the one with additional attenuation (Schelkshorn, 2006).

To be able to compare the performance of the four mentioned methods a simulation was carried out where the object was placed at several different positions and the initial value for the unknowns used for the linearization was kept constant. Figure 3 shows a comparison of the results as a histogram. The four methods are designated as follows: 


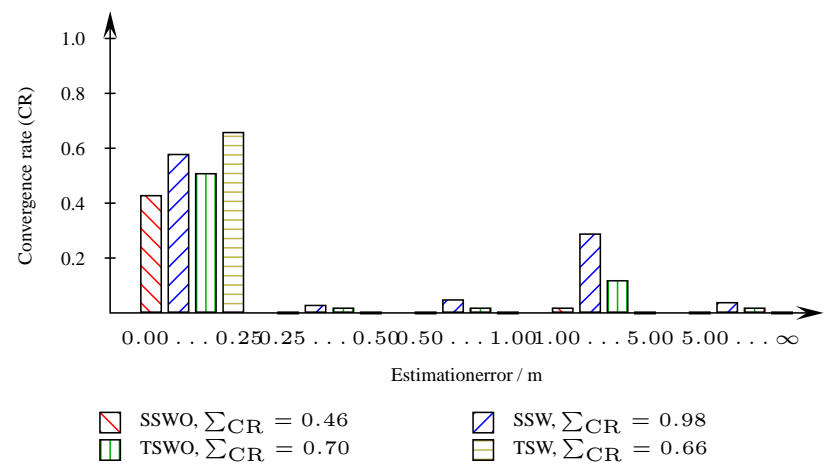

Fig. 3. Convergence rate and error with different algorithms.

- Single step without attenuation (SSWO): standard Newtons Method

- Single step with attenuation (SSW): Newtons Method with additional attenuation

- Two step without attenuation (TWSO): Newtons Method without attenuation, combined for two timesteps

- Two step with attenuation (TSW): Newtons Method with attenuation, combined for two timesteps

As it is possible to detect whether the solution converges or not, but wrong results can not be detected, one should use the ratio of the number of correct results versus the number of wrong results as a figure of merrit.

One can see that introducing the attenuation yields a higher convergence rate but also a higher number of wrong results. Adding the information from the second timestep also increases the convergence rate compared to the standard Newtons Method with a lower number of wrong results compared to the attenuated version. Finally combining both, a second timestep and the attenuation turns out to be the best alternative, as there are almost no wrong results.

\section{Multi target environments}

Independent of the method of solving the resulting system of equations the whole principle is based on doppler measurement. This doppler measurement can easily be implemented in existing RF-infrastructure. As the system of equations relys on the combination of four doppler sensors one has to assure that each sensor observes only one doppler signal. That means in this simple setup only single target scenarios can be handled.

To deal with multi target scenarios it is necessary to separate the occuring doppler signals before further processing. After separation the described system of equations can be solved for each set of doppler signals.
Table 1. 4-Ch. Radar, System parameters.

\begin{tabular}{ll}
\hline Parameter & Value \\
\hline Number of channels & 4 \\
Center frequency & $2.45 \mathrm{GHz}$ \\
Radar modes & $\mathrm{CW}, \mathrm{FSK}, \mathrm{SFCW}$ \\
Max. bandwidth & $600 \mathrm{MHz}$ \\
Sweeptime (SFCW) & $5 \mathrm{~ms}$ \\
Max. sampling frequency & $25 \mathrm{kHz}$ \\
Output power & $15 \mathrm{dBm}$ \\
\hline
\end{tabular}

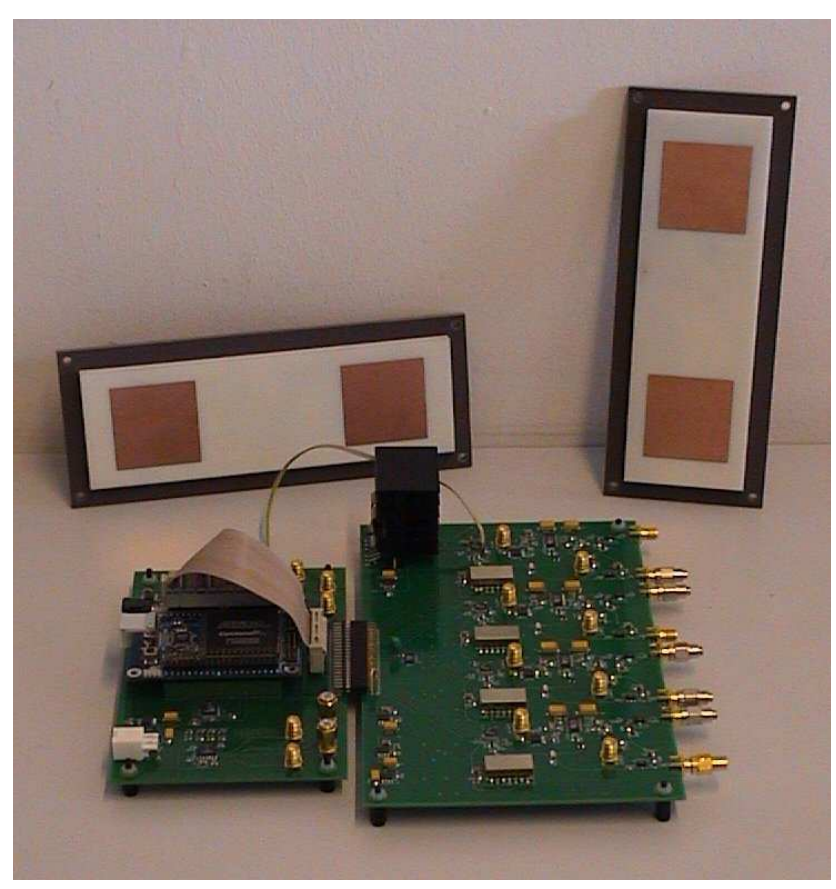

Fig. 4. 4-Ch. CW/FSK/SFCW-Radar @ $2.45 \mathrm{GHz}$.

\section{Demonstration system}

To verify the simulation results obtained so far, a demonstration system at $2.45 \mathrm{GHz}$ is setup at the moment. This demonstration system consists of four identical channels with a central control unit based on an FPGA-Evaluation board. Each channel mainly consists of a PLL for signal generation and an I/Q-mixer in the receiver section. The I/Q-channels then are $\mathrm{A} / \mathrm{D}$-converted and the resulting data is transferred via LAN to a $\mathrm{PC}$ for processing. By continuously reprogramming the four PLLs it is also possible to generate modulated signals. So far FSK- and SFCW-modulation is considered additionally to the simple $\mathrm{CW}$ operation.

All relevant system parameters are summarized in Table 1. A photo of the actual design is shown in Fig. 4. 


\section{Conclusions}

The presented setup is an easy way of position finding especially for indoor applications where other methods (e.g. GPS) won't work. Due to the simple approach of doppler measurement it can easily be implemented in already existing RF-infrastructure. In addition to the position information of the unkown object also its velocity is obtained. A notably advantage of this approach is that no active participation of the unknown object is required.

As this approach relys on the measurement of doppler signals it is only applicable in enviroments with moving targets.

\section{References}

Hettwer, J. and Benning, W.: Erweiterung des Konvergenzbereichs bei nichtlinearen Ausgleichungsaufgaben, Allgemeine Vermessungs-Nachrichten, 254-259, 2001.

Schelkshorn, S.: Konvergenzuntersuchung: Lösungsverfahren für Vier-Sensor-System, Tech. rep., TU München, Lehrstuhl für Hochfrequenztechnik (HFS), 2006.

Werner, J.: Numerische Mathematik, Bd. 1, Lineare und nichtlineare Gleichungssysteme, Interpolation, numerische Integration, Vieweg, 1992. 